\title{
Efficacy and safety of artemisinin-based combination therapy, and molecular markers for artemisinin and piperaquine resistance in Mainland Tanzania
}

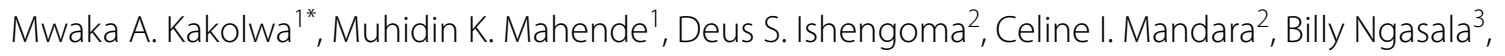
Erasmus Kamugisha ${ }^{4}$, Johannes B. Kataraihya ${ }^{4}$, Renata Mandike ${ }^{5}$, Sigsbert Mkude ${ }^{5}$, Frank Chacky ${ }^{5}$, Ritha Njau ${ }^{6}$, Zul Premji ${ }^{7}$, Martha M. Lemnge ${ }^{2}$, Marian Warsame ${ }^{8}$, Didier Menard ${ }^{9}$ and Abdunoor M. Kabanywanyi ${ }^{1}$

\begin{abstract}
Background: Artemisinin-based combination therapy (ACT) is the first-line anti-malarial treatment of uncomplicated malaria in most malaria endemic countries, including Tanzania. Unfortunately, there have been reports of artemisinin resistance and ACT failure from South East Asia highlighting the need to monitor therapeutic efficacy of ACT in these countries as recommended by World Health Organization.

Methods: Open-label single arm studies in mainland Tanzania were conducted in nine sentinel sites in 2011, 2012 and 2015 to assess the efficacy and safety of artemether/lumefantrine (AL) and artesunate/amodiaquine (ASAQ) using 28 days follow-up and dihydroartemisinin/piperaquine (DHAPQ) using 42 days follow-up. Mutations in the propeller domain of the Plasmodium falciparum kelch 13 (k13) gene and amplification of the P. falciparum plasmepsin 2(pm2) gene, associated with artemisinin and piperaquine $(\mathrm{PQ})$ resistance, were also investigated.

Results: Of the 428 patients enrolled, 328 patients provided study endpoint. For AL, the PCR corrected per-protocol analysis showed adequate clinical and parasitological response (ACPR) of $90.3 \%(n=28 ; 95 \% \mathrm{Cl} 74.2-98.0)$ in Kyela $2012,95.7 \%(n=22 ; 95 \%$ Cl 78.1-99.0) in Chamwino, 100\% in Muheza ( $n=29 ; 95 \%$ Cl 88.1-100), 100\% in Nagaga $(n=39 ; 95 \% \mathrm{Cl} 91.0-100)$ and Kyela $2015(n=60 ; 95 \% \mathrm{Cl} 94.0-100)$. For ASAQ, PCR corrected ACPR of $98 \%(n=49$; 95\% Cl 89.4-99.9) and 100\% ( $n=25 ; 95 \% \mathrm{Cl} 86.3-100)$ were observed in 2011 in Ujiji and Kibaha, respectively. For DHAPQ, the ACPR was $100 \%(n=71 ; 95 \% \mathrm{Cl} 94.9-100)$. Of the 235 samples with genetic interpretable results, only 7 (3\%) had non-synonymous k13 mutations. None of these are candidate or validated markers of artemisinin resistance and all patients carrying these alleles cleared the parasites on day 3. Of the DHAPQ group, 10\% (3/29) of the samples with interpretable results had pm2 multiple copies and none of them was associated with treatment failure.
\end{abstract}

Conclusion: All the tested ACT in mainland Tanzania were highly efficacious and none of validated $k 13$ mutants associated with artemisinin resistance was observed. However, three isolates with multiple copy numbers of $p m 2$ gene associated with PQ resistance among the limited samples tested successfully calls for further investigation.

Trial registration Number ACTRN12615000159550. Registered 18th February 2015, https://www.anzctr.org.au/trial/ MyTrial.aspx

Keywords: Artemisinin-based combination therapy, Efficacy, Safety, Malaria, Molecular markers, Artemisinin, Piperaquine, Tanzania

\footnotetext{
*Correspondence: mkakolwa@ihi.or.tz

${ }^{1}$ Ifakara Health Institute, Dar es Salaam, Tanzania

Full list of author information is available at the end of the article
} 


\section{Background}

Despite the global fall of malaria cases over the past decade, the disease still caused 6.8 million cases and 18,930 deaths in Tanzania in 2016 [1]. Malaria affects different age groups with children below 5 years carrying most of the burden [1]. Plasmodium falciparum is the predominant malaria species. Effective case management-early diagnosis and prompt treatment-is one of the pillars of malaria control and elimination. Following the World Health Organization (WHO) recommendation of artemisinin-based combination therapy (ACT) for the treatment of uncomplicated malaria, the Ministry of Health, Community Development, Gender, Elderly and Children of Tanzania introduced artemether/lumefantrine $(\mathrm{AL})$ as first-line treatment for uncomplicated falciparum malaria in 2006 [2].

The emergence and spread of artemisinin resistance, as well as increased treatment failure $(>10 \%)$ with ACT in parts of South East Asia, underscores the need for malaria endemic countries to be vigilant and continuously evaluate the efficacy of the ACT medicines being used $[3,4]$. WHO recommends monitoring the efficacy of the nationally used ACT every 2 years to inform national anti-malarial treatment policy to ensure effective treatment of malaria patients [5].

A review of nine studies previously conducted in different areas of Tanzania (in Muheza, Bagamoyo, Kibaha, Tabora, Mwanza, Kilombero, Zanzibar and Kyela) from 2002 to 2013 reported PCR corrected cure rate ranging from 88 to $94 \%$ for artesunate/amodiaquine (ASAQ) and 91 to $100 \%$ for AL. Only two studies reported cases of positive parasitaemia on day 3 which was below 2\% [6]. Studies from East Africa and other parts of Africa also reported a high efficacy of ACT [7-15].

In vivo assessment of therapeutic efficacy of antimalarial medicine is the gold standard for generating information to inform national malaria treatment policy. In addition detection of mutations or amplifications in Plasmodium falciparum genes linked to anti-malarial drug resistance confirms parasite resistance, supplementing the in vivo efficacy data. The discovery of point mutations in the pfkelch13 (k13) gene and amplification of pfplasmepsin 2 gene provide opportunities to monitor artemisinin and piperaquine resistance, respectively $[16,17]$.

The current paper reports the findings of therapeutic efficacy studies conducted from 2011 to 2015, assessing the therapeutic efficacy and safety of AL, ASAQ and dihydroartemisinin/piperaquine (DHAPQ) in the mainland Tanzania.

\section{Methods}

Study design and areas

Open-label, one-arm prospective studies $(\mathrm{N}=8)$ were conducted to evaluate the clinical and parasitological responses to directly observed treatment of $\mathrm{AL}$ and ASAQ in 2011, AL in 2012, and AL and DHAPQ in 2015 using the WHO protocol for the surveillance of the efficacy of anti-malarial drugs [18]. The studies were conducted between March and September, covering the peak transmission season (June-August). They were carried out in the nine National Malaria Control Programme (NMCP) sentinel sites in eight regions; Chamwino in Dodoma, Butimba in Mwanza, Kibaha and Rufiji in Pwani, Kyela in Mbeya, Kilombero in Morogoro, Muheza in Tanga, Nagaga in Mtwara, and Ujiji in Kigoma (Fig. 1). Type of anti-malarial tested per site was agreed upon by all investigators ahead of protocol's ethics approvals. In 2011 ASAQ was evaluated in Ujiji and Kibaha and AL in Kilombero and Muheza. Due to low number of patients enrolled in 2011, the Tanzania first-line anti-malarial (AL) was recommended to be assessed at all four sites (Butimba, Chamwino, Nagaga and Kyela) in 2012 while in 2015, AL and DHAPQ were evaluated only in Kyela and Rufiji, respectively.

\section{Participants and enrolment}

Patients were enrolled if they had mono-infection of P. falciparum detected by microscopy, axillary temperature $\geq 37.5{ }^{\circ} \mathrm{C}$ or history of fever during the past $24 \mathrm{~h}$, ability to swallow oral medication, ability and willingness to comply with the study protocol and visit schedule for the duration of the study and informed consent from the parent or guardian of the enrolled children. The age of the patients enrolled varied slightly in the different years: 6-59 months in 2011; 6 months -10 years in 2012 and 6 months and above in 2015. Similarly, the threshold of parasitaemia at enrolment varied from 1000 to 200,000 asexual/ $\mu$ l asexual forms in 2011 and in 2012 to $250-200,000$ asexual/ $\mu \mathrm{l}$ in 2015. WHO protocol allows to adjust age and enrolment parasitaemia of study patients according to the transmission levels [18]. ACT is contraindicated in the first trimester of pregnancy and, therefore, the WHO protocol requires that all females who reached menarche to be tested for pregnancy. For the study in 2015 female minors aged 12-17 years were excluded from the study as confidentiality and privacy required for pregnancy testing for this age group could not be ensured. Any of the following features was regarded as an exclusion criteria: presence of general danger signs or signs of severe falciparum malaria according to the definitions of WHO, severe malnutrition, febrile 


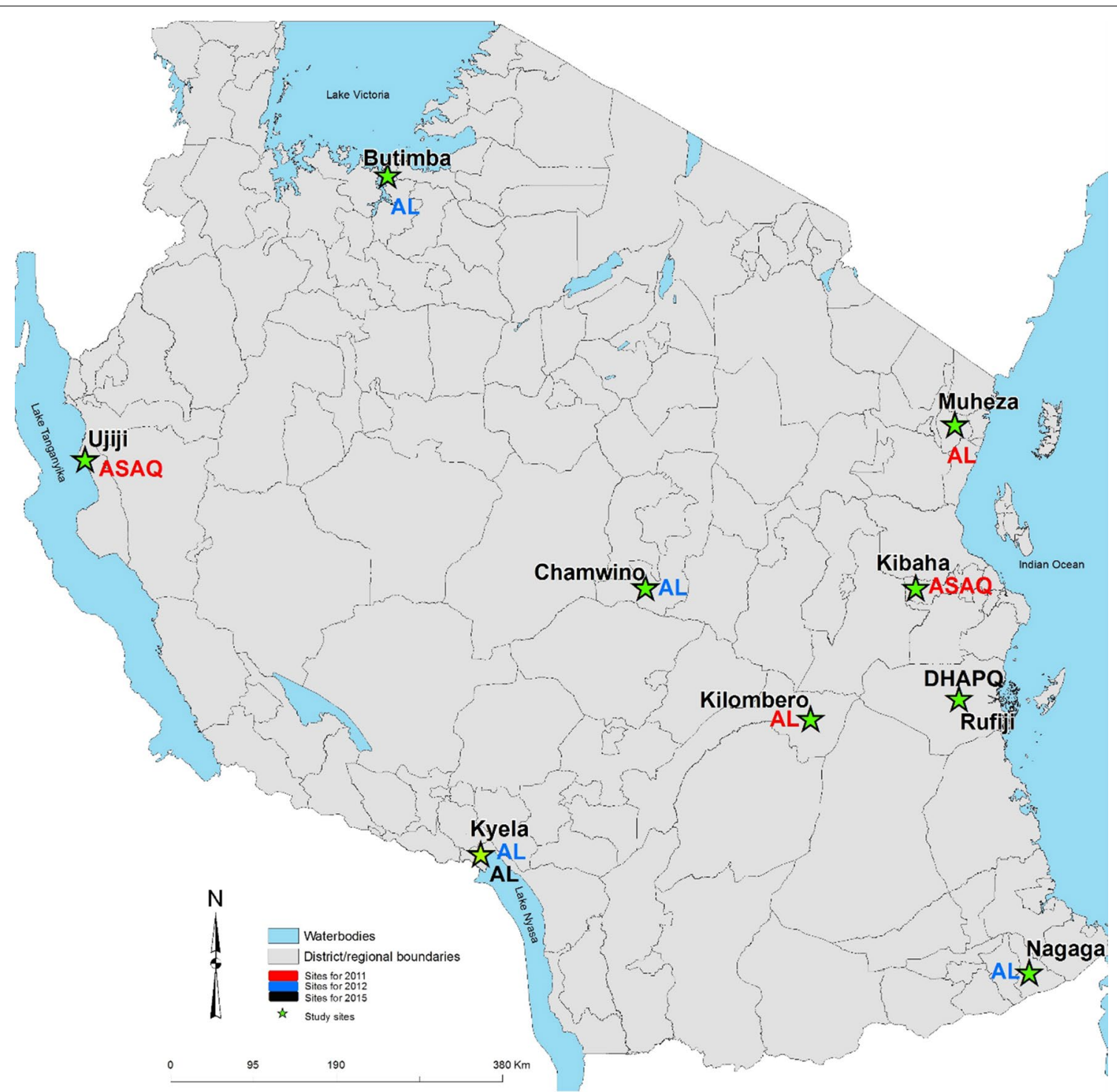

Fig. 1 Map of Mainland Tanzania showing the location of the studies conducted in 2011 (red), in 2012 (blue) and in 2015 (black) by ACT treatment

conditions due to diseases other than malaria or other known underlying chronic or severe diseases, regular medication, which may interfere with anti-malarial treatment, history of hypersensitivity reactions or contraindications to any of the medicine(s) being tested or used as alternative treatment(s).

At the enrollment, home address, demographic information and full medical history were taken from each patient. Body weight was measured and axillary temperature was taken. Physical examination was done to rule out danger signs and other non-malaria illnesses as per exclusion criteria.

\section{Treatments and follow-up}

All eligible patients were treated with oral AL, ASAQ or DHAPQ using the WHO recommended therapeutic dose regimens [19]. All treatment doses were given under direct supervision of the study clinician or study nurse in health facilities and participants were observed for $30 \mathrm{~min}$ for adverse reactions or vomiting. For AL arm, participants residing far from the health facilities were retained at the facility for the night doses and allowed to go home in the morning. The daily dose was re-administered in case of vomiting within $30 \mathrm{~min}$ after drug intake, one child was withdrawn from the study and given the 
rescue medication after vomiting the replacement dose. Children with fever $\geq 38.5{ }^{\circ} \mathrm{C}$ were treated with paracetamol and tepid sponging was also used for those below 5 years. Patients were assessed clinically and parasitology according to the WHO protocol [18] and were followed up for 28 days for treatment with AL and ASAQ, and 42 days for DHAPQ.

\section{Laboratory assessment}

Thick and thin smears were taken on days 0 (before treatment) and during subsequent prescribed follow-up on day 1 (if presented with danger signs or severe symptoms), $2,3,7,14,21,28$ days in 28 days for AL and ASAQ whereas for DHAPQ up to day 42 days. Microscopy examination of thick and thin blood films using Giemsa stain was undertaken to determine parasite species and density according to the WHO protocol [18]. Parasite density (per $\mu \mathrm{l}$ ) was calculated assuming a white blood cell count of $8000 / \mu \mathrm{l}$. All slides were read independently by two qualified microscopists and the average of the two counts was taken as the parasite density. Parasite counts with discordant results (differences in species, presence of parasites or parasite density $>50 \%$ ) were re-read by a third independent microscopist, and parasite densities were calculated by averaging the two most close counts.

Two to three drops of blood were collected on filter paper (Whatman no. $3 \mathrm{~mm}$, GE Healthcare UK Limited, UK) on day 0 (before treatment) and during recurrence of parasitaemia on day 7 onwards. Each dried blood spots (DBS) was punched with a sterile puncher and placed in a 96 well plate in numerical order. Samples were lysed overnight in a saponin solution, and then, DNAs were extracted with the Instagen Matrix resin as previously described [20]. All DNA samples from patients undergoing symptoms reoccurrence were analysed for genotyping of the highly polymorphic regions $m s p 1, m s p 2$ and glurp loci, as recommended by WHO [21]. DNA samples were also analysed for the presence of mutations in the propeller domain in the $k 13$ gene associated to artemisinin resistance [16]: a portion of the $k 13$ gene was amplified using a nested PCR assay, amplicons (codons 443-666, i.e. 720 bp) were sent to Macrogen Korea for sequencing, and DNA sequences were analysed to identify specific single-nucleotide polymorphism (SNP) related to artemisinin resistance. Samples from the DHAPQ treated patients were also analysed for amplification of plasmepsin 2 gene recently associated to piperaquine resistances [17].

\section{Treatment outcome}

Treatment outcomes were determined according to the classification of WHO [18] as early treatment failure (ETF), late clinical failure (LCF), late parasitological failure (LPF) and adequate clinical and parasitological response (ACPR). The proportion of patients who were still positive on day 3 was also recorded. Following PCR correction, treatment failure was classified as recrudescence if recurrent parasitaemia was the same parasite strain as of day 0 or new infection if they were different parasite strain. Adverse events including serious adverse events were assessed through physical examination and history taking.

\section{Sample size and statistical analysis}

An expected treatment failure rate of $5 \%$ was assumed for all the three drugs evaluated and with a $95 \%$ confidence level and a precision of 5\%, a sample size of 73 was targeted per site or per drug for 2011, 2012 and 2015 studies. An additional $20 \%$ was added to ensure that the sample size would be achieved after the exclusion of patients due to loss to follow-up or withdrawal. A total of 88 patients per drug per site were therefore targeted. Data were double entered and analysed using the WHOdesigned Microsoft Excel ${ }^{\circledR}$ program (Microsoft Corporation, Washington). Chi-square test or the Fisher's exact test (where cell counts were $\leq 5$ ) were used for comparing differences in categorical data. Arithmetic and geometric (only for parasite density) means were considered.

Proportions of treatment failures (ETF, LCF and LPF) and cure rates before and after PCR correction were calculated as per-protocol analysis and Kaplan-Meier analysis. Re-infections and non-interpretable PCR results were excluded from the per-protocol analysis, but retained in the Kaplan-Meier analysis.

\section{Ethics approvals and registration}

These studies were approved by the Ifakara Health Institute (IHI) ethics review committee (ERC), the National Medical Research Coordinating Committee (MRCC) of the National Institute for Medical Research (NIMR) and by the WHO research ethics review committee for studies conducted in 2015.

All English consent forms were translated into Swahili and back translated by a native speaker. Before enrollment, parents or guardians of children below 18 years were asked for a written informed consent. In addition, an assent was sought for all children between 12 and 17 years. For illiterate parents or guardians, a literate witness who was not part of the study team was chosen to sign on his or her behalf. All the information obtained was kept confidential and shared with study team only when it was necessary. Identification numbers were used instead of patients' names in the case report forms and computer based data entry. 
Table 1 Baseline characteristics of the study cases at enrollment from the different sites

\begin{tabular}{|c|c|c|c|c|c|c|}
\hline Year & Drug/site & $\begin{array}{l}\text { Enrolled } \\
\text { numbers }\end{array}$ & $\begin{array}{l}\text { Age (years) } \\
\text { Median (range) }\end{array}$ & $\begin{array}{l}\text { Sex (male) } \\
\text { n (\%) }\end{array}$ & $\begin{array}{l}\text { Temperature }\left({ }^{\circ} \mathrm{C}\right) \\
\text { Mean (SD) }\end{array}$ & $\begin{array}{l}\text { Parasitaemia ( } \mu \mathrm{l}) \\
\text { GM (range) }\end{array}$ \\
\hline \multirow[t]{5}{*}{2011} & ASAQ & & & & & \\
\hline & Ujiji & 73 & $2.7(0.2-5)$ & $37(50.7)$ & $38.4(1.3)$ & $48,183(1600-198,194)$ \\
\hline & Kibaha & 29 & $3.0(1-5)$ & $17(58.6)$ & $38.2(1.1)$ & $6162(1034-80,000)$ \\
\hline & $\mathrm{AL}$ & & & & & \\
\hline & Muheza & 32 & $2.0(1-5)$ & $18(56.3)$ & $37.9(1.2)$ & $24,400(1680-160,457)$ \\
\hline \multirow[t]{4}{*}{2012} & $\mathrm{AL}$ & & & & & \\
\hline & Chamwino & 26 & $5.0(1-8)$ & $13(50.0)$ & $38.1(1)$ & $8146(1120-36,800)$ \\
\hline & Kyela & 44 & $3.0(1-10)$ & $20(45.5)$ & $38.1(1.2)$ & $32,741(1680-180,800)$ \\
\hline & Nagaga & 62 & $3.0(1-10)$ & $30(48.4)$ & $38.3(0.9)$ & $22,368(1040-149,382)$ \\
\hline \multirow[t]{4}{*}{2015} & $\mathrm{AL}$ & & & & & \\
\hline & Kyela & 80 & $6.5(0.6-60)$ & $40(50.0)$ & $38.1(1.3)$ & $13,303(640-84,480)$ \\
\hline & DHA-PQ & & & & & \\
\hline & Rufiji & 82 & $10(0.8-87)$ & $44(51.2)$ & $37.6(0.9)$ & $12,007(960-199,120)$ \\
\hline
\end{tabular}

GM geometric mean, $A S A Q$ artesunate/amodiaquine, $A L$ artemether/lumefantrine, $D H A-P Q$ dihydroartemisinin/piperaquine

\section{Results}

The baseline characteristics of all study patients are summarized in Table 1. A total of 3980 patients were screened and only 428 (11\%) met criteria for enrollment (Fig. 2). There was no significant difference in sex and body temperature while there was variation of geometric mean of parasite density across the sites. In addition, the age profile of the study patients varied between the years ranging from 5 years in 2011, up to 10 years in 2012 and all ages in 2015 based on the inclusion criteria for each year.

Tables 2 and 3 present the treatment outcomes by year, site and drug. A total of 64 patients were lost during the follow-up, 18 re-infections and 5 were excluded after enrolment due to consent withdrawal. PCR non-corrected ACPR varied from 75 to $100 \%$ and 78 to $100 \%$ for ASAQ and AL, respectively (Table 2), while $99 \%$ of the patients treated with DHAPQ achieved ACPR. No ETF was observed. All study patients cleared their parasites by day 3 except two cases (6.3\%) treated with AL in Muheza. PCR corrected ACPR was $98 \%$ and above for ASAQ, 90\% and above for AL and 100\% for DHAPQ (Table 3).

\section{K13 mutations}

Among 428 recruited patients, 324 day 0 samples were available and analysed for $k 13$ mutations. Out of these, 235 samples gave interpretable sequence and the remaining 89 samples were either PCR negative $(n=51)$ or gave non-interpretable data $(\mathrm{n}=38)$. Out of the samples with interpretable data, $93.6 \%(n=220)$ had wild-type $k 13$ alleles. The remaining carried, non-synonymous $k 13$ mutations $(3.0 \%, \mathrm{n}=7)$ and synonymous $k 13$ mutations $(3.4 \%, \mathrm{n}=8)$. The non-synonymous $k 13$ mutants observed were L463S, G496S, M476I, V510M, E556K, M562T and E602D. None of them was associated with day 3 positive parasitaemia and they are not among the known $k 13$ mutants associated with artemisinin resistance. Of note, the M476I mutant, previously associated with in vitro artemisinin resistance in a similar Tanzania strain (F32-ART) was observed once in 2012 in a patient treated with AL from Kyela site. Though the patient was lost follow up on day 21, all the parasites were cleared up by day 3 .

\section{Plasmepsin2 gene amplification}

All the DHAPQ (82/82) D0 samples were available for pm2 gene amplification analysis. Of these, 29 (35.4\%) samples gave interpretable data of which $3(10.3 \%)$ had two copies pm2 and all had ACPR. The sequences of the remaining $53(64.6 \%)$ samples gave non-interpretable results due to too low amount of DNA or poor quality of DNA extracts.

\section{Adverse events}

Few non-serious adverse events were reported across the sites, the most common were cough $23 / 428$ (5.4\%), vomiting $10 / 428(2.3 \%)$, fever $4 / 428(0.9 \%)$ and diarrhoea $4 / 428(0.9 \%)$. One participant from Chamwino vomited AL twice, he was withdrawn from the study and was given quinine injection as a rescue treatment. A death of a female child aged 6 years from Nagaga site was reported. The information regarding the possible cause of death was not known, the child attended the first two visits only. 


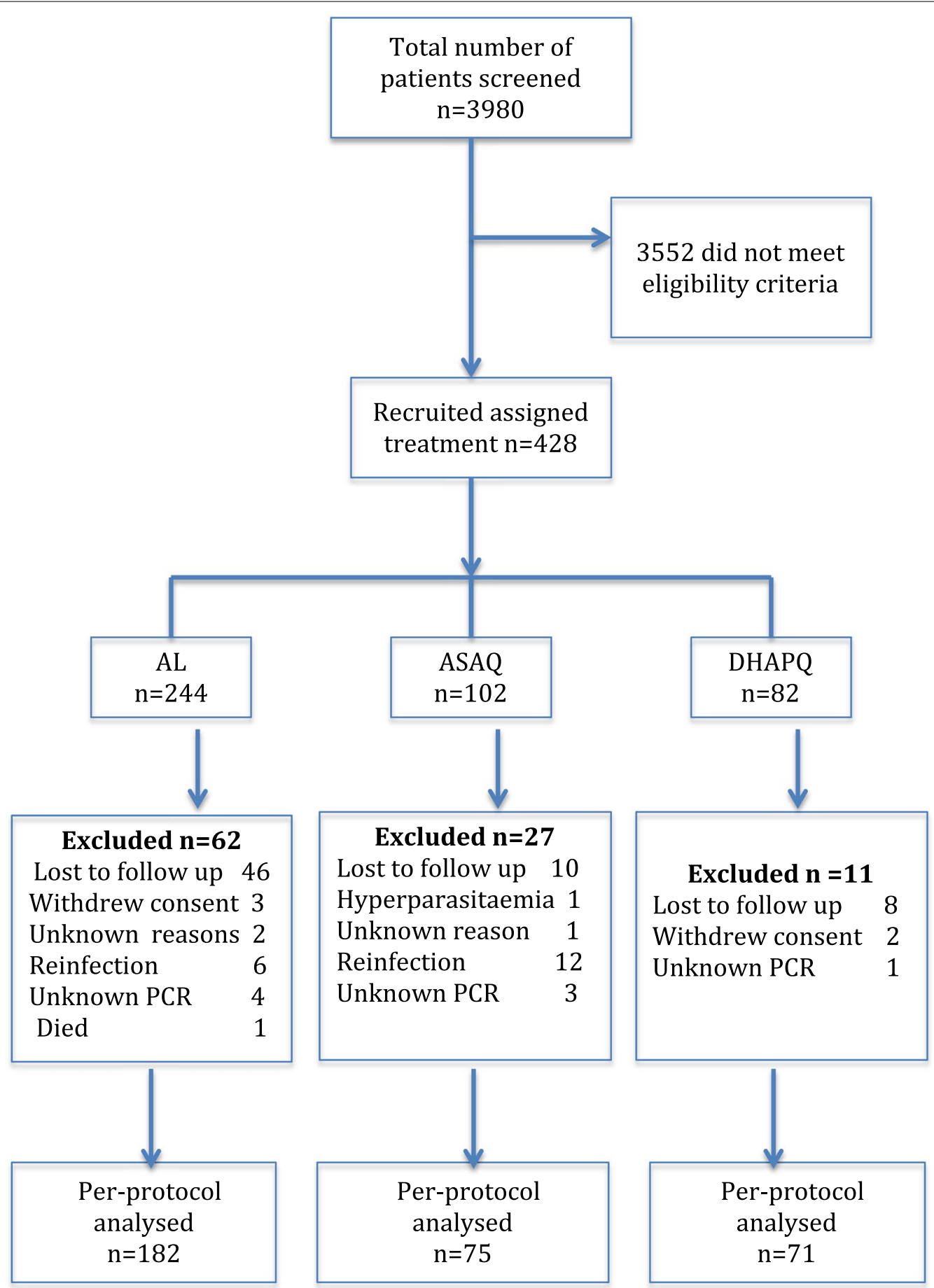

Fig. 2 Flow chart: $A S A Q$ artesunate + amodiaquine, $A L$ artemether + lumefantrine, $D H A P Q$ dihydroartemisinin + piperaquine

\section{Discussion}

The findings showed PCR-corrected cure rate of $90 \%$ and above for AL, $98 \%$ and above for ASAQ and $100 \%$ for DHAPQ treatments in the study areas between 2011 and 2015. Treatment with AL, the first-line drug for treatment of uncomplicated falciparum malaria in Mainland Tanzania, achieved ACPR above 95\% except in 2012 in Kyela where ACPR was $90 \%$ the borderline below which WHO recommends ACT should be replaced [19]. However, the result from the same site 3 years later showed cure rate 
Table 2 Treatment responses in patients treated with artesunate/amodiaquine, artemether/lumefantrine and dihydroartemisinin/piperaquine in Tanzania: PCR uncorrected

\begin{tabular}{|c|c|c|c|c|c|c|c|c|}
\hline Year & Drug/site & $\mathrm{N}$ & $\begin{array}{l}\text { PD3 } \\
\text { n (\%) }\end{array}$ & $\begin{array}{l}\text { Excluded/lost } \\
\mathrm{n}(\%)\end{array}$ & $\begin{array}{l}\text { LCF } \\
\%(95 \% \mathrm{Cl})\end{array}$ & $\begin{array}{l}\text { LPF } \\
\%(95 \% \mathrm{Cl})\end{array}$ & $\begin{array}{l}\text { ACPR } \\
\%(95 \% \mathrm{Cl})\end{array}$ & $\begin{array}{l}\text { Kaplan-Meier } \\
\%(95 \% \mathrm{Cl})\end{array}$ \\
\hline \multirow[t]{5}{*}{2011} & ASAQ & & & & & & & \\
\hline & Ujiji & 73 & 0 & $8(11.0)$ & $13.8(6.5-24.7)$ & $10.8(4.4-20.9)$ & $75.4(63.1-85.2)$ & $76.1(64.0-84.6)$ \\
\hline & Kibaha & 29 & 0 & $4(13.8)$ & $0(0.0-13.7)$ & $0(0.0-13.7)$ & $100(86.3-100)$ & 100 \\
\hline & $\mathrm{AL}$ & & & & & & & \\
\hline & Muheza & 32 & $2(6.3)$ & $2(6.3)$ & $0(0.0-11.6)$ & $3.3(0.1-17.2)$ & 96.7 (82.8-99.9) & 96.9 (79.8-99.6) \\
\hline \multirow[t]{4}{*}{2012} & $\mathrm{AL}$ & & & & & & & \\
\hline & Chamwino & 26 & 0 & $3(11.5)$ & $0(0.0-14.8)$ & $4.3(0.1-21.9)$ & 95.7 (78.1-99.9) & 95.7 (72.9-99.4) \\
\hline & Kyela & 44 & 0 & $8(18.2)$ & $5.6(0.7-18.7)$ & $16.7(6.4-32.8)$ & $77.8(60.8-89.9)$ & $78.9(62.3-88.9)$ \\
\hline & Nagaga & 62 & 0 & $23(37.1)$ & $0(0.0-9.0)$ & $0(0.0-9.0)$ & $100(91.0-100)$ & 100 \\
\hline \multirow[t]{4}{*}{2015} & $\mathrm{AL}$ & & & & & & & \\
\hline & Kyela & 80 & 0 & $16(20.0)$ & $4.7(1.0-13.1)$ & $1.6(0.0-8.4)$ & $93.8(84.8-98.3)$ & $94.1(85.0-97.8)$ \\
\hline & DHA-PQ & & & & & & & \\
\hline & Rufiji & 82 & 0 & $10(12.2)$ & $1.4(0.0-7.5)$ & $0(0.0-5.0)$ & $98.6(92.5-100)$ & 98.6 (90.8-99.8) \\
\hline
\end{tabular}

$P D 3$ positive on day 3, $L C F$ late clinical failure, $L P F$ late parasitological failure, $A C P R$ adequate clinical and parasitological response, $A S A Q$ artesunate + amodiaquine, $A L$ artemether + lumefantrine, $D H A P Q$ dihydroartemisinin + piperaquine

Table 3 Treatment responses in patients treated with artesunate/amodiaquine, artemether/lumefantrine and dihydroartemisinin/piperaquine in Tanzania: PCR corrected

\begin{tabular}{|c|c|c|c|c|c|c|c|c|c|}
\hline Year & Drug/site & $\mathbf{N}$ & $\begin{array}{l}\text { Excluded/lost } \\
\text { n (\%) }\end{array}$ & $\begin{array}{l}\text { Re-infection } \\
\text { n (\%) }\end{array}$ & $\begin{array}{l}\text { Uknown } \\
\text { n (\%) }\end{array}$ & $\begin{array}{l}\text { LCF } \\
\%(95 \% \mathrm{CI})\end{array}$ & $\begin{array}{l}\text { LPF } \\
\%(95 \% \mathrm{Cl})\end{array}$ & $\begin{array}{l}\text { ACPR } \\
\%(95 \% \mathrm{Cl})\end{array}$ & $\begin{array}{l}\text { Kaplan-Meier } \\
\%(95 \% \mathrm{Cl})\end{array}$ \\
\hline \multirow[t]{5}{*}{2011} & ASAQ & & & & & & & & \\
\hline & Ujiji & 73 & $8(11.0)$ & $12(16.4)$ & $3(4.1)$ & $0(0.0-7.1)$ & $2(0.1-10.6)$ & 98 (89.4-99.9) & 98.3 (88.8-99.8) \\
\hline & Kibaha & 29 & $4(13.8)$ & $\mathrm{NA}$ & NA & $0(0.0-13.7)$ & $0(0.0-13.7)$ & $100(86.3-100)$ & 100 \\
\hline & $\mathrm{AL}$ & & & & & & & & \\
\hline & Muheza & 32 & $2(6.3)$ & 0 & $1(3.1)$ & $0(0.0-11.9)$ & $0(0.0-11.9)$ & $100(88.1-100)$ & 100 \\
\hline \multirow[t]{4}{*}{2012} & $\mathrm{AL}$ & & & & & & & & \\
\hline & Chamwino & 26 & $3(11.5)$ & 0 & 0 & $0(0.0-14.8)$ & $4.3(0.1-21.9)$ & $95.7(78.1-99.0)$ & 95.7 (72.9-99.4) \\
\hline & Kyela & 44 & $8(18.2)$ & $3(6.8)$ & $2(4.5)$ & $6.5(0.8-21.4)$ & $3.2(0.1-16.7)$ & $90.3(74.2-98.0)$ & $91.5(75.9-97.2)$ \\
\hline & Nagaga & 62 & $23(37.1)$ & NA & NA & $0(0.0-9.0)$ & $0(0.0-9.0)$ & $100(91.0-100)$ & 100 \\
\hline \multirow[t]{4}{*}{2015} & $\mathrm{AL}$ & & & & & & & & \\
\hline & Kyela & 80 & $16(20.0)$ & $3(3.8)$ & $1(1.3)$ & $0(0.0-6.0)$ & $0(0.0-6.0)$ & $100(94.0-100)$ & 100 \\
\hline & DHA-PQ & & & & & & & & \\
\hline & Rufiji & 82 & $10(12.2)$ & 0 & $1(1.2)$ & $0(0.0-5.1)$ & $0(0.0-5.1)$ & $100(94.9-100)$ & 100 \\
\hline
\end{tabular}

Unknown unknown based on PCR analysis, $L C F$ late clinical failure, $L P F$ late parasitological failure, $A C P R$ adequate clinical and parasitological response, $A S A Q$ artesunate/amodiaquine, $A L$ artemether/lumefantrine, $D H A P Q$ dihydroartemisinin/piperaquine

of $100 \%$ for the same ACT medicine. Since all patients, including under-five children $(\mathrm{n}=35)$, achieved ACPR in 2015, the higher treatment response in 2015 could not be attributed to the inclusion of older children $(n=34)$ and adults $(n=11)$, population groups with potentially higher immunity to enhance treatment outcome.

$\mathrm{AL}$ is the first-line treatment of choice in most of the malaria endemic African countries and has remained highly effective after more than a decade of its use [7-15].
In Tanzania AL is still highly efficacious despite the fact that mutations in $m d r 1$ codons have been detected and showed increased trend [22, 23].

Anti-malarial ASAQ is not included in the national first-line treatment policy of the mainland Tanzania, although it is widely available in the private sector and is the first-line treatment in Zanzibar [1], which is part of the United Republic of Tanzania. This study gave reassurance that this ACT medicine is highly efficacious for the 
treatment of uncomplicated falciparum malaria. Similar high cure rate has been reported from other African countries [7, 9, 12, 14, 15, 24, 25].

The high cure rate of DHAPQ in this study supports earlier findings from and across Africa [11, 15, 24, 26-28]. This ACT medicine is being increasingly recommended in malaria endemic countries as secondline treatment for falciparum malaria and also used for mass drug administration in special situations. However, the emergence of piperaquine resistance as well as high treatment failure with DHAPQ from several countries in South East Asia calls for continuous monitoring in countries using this ACT.

Suspected artemisinin resistance is defined as $\geq 10 \%$ of patients with persistent parasitaemia by microscopy at $72 \mathrm{~h}$ (day 3) after treatment with ACT or artesunate monotherapy or $\geq 10 \%$ of patients with a half-life of the parasite clearance slope $\geq 5 \mathrm{~h}$ after treatment with ACT or artesunate monotherapy or $\geq 5 \%$ of patients carrying k13 resistance-confirmed mutations [29]. Artemisinin resistance is confirmed if $\geq 5 \%$ of patients carrying $k 13$ resistance-confirmed mutations and that patients have either persistent parasitaemia by microscopy on day 3 after treatment with ACT or artesunate monotherapy, or a half-life of the parasite clearance slope $\geq 5 \mathrm{~h}$ [29]. In the current study, the lack of $k 13$ validated mutants [29] supported by the clearance of parasitaemia by day 0 in all patients might suggest absence of suspected artemisinin resistance in $P$. falciparum in the study areas. This finding is in line with previous report from Africa [30]. So far, several non-synonymous $k 13$ alleles have been reported in different parts of Africa, none of which has been linked to alleles from South-East Asia neither associated with day 3 parasitaemia or delayed parasite clearance [31-34]. The M476I mutant, found in one patient from Kyela in 2012, was not observed in studies conducted the same site in 2015.

The recent evidence provided by Witkowski and colleagues [17] and Amato et al. has shown a high association between the pm 2 multiple copies and piperaquine resistance in Cambodia [35]. DHAPQ treatment failure, associated with increased $p m 2$ multiple copies, was reported from several countries in South East Asia [17]. The findings of $10 \%$ cases with $p m 2$ copies $(\mathrm{n}=2)$ among the limited samples $(n=30)$ successfully analysed, indicate the possibility of presence of parasites resistant to piperaquine in Tanzania and calls for further studies with adequate sample size. Recently low prevalence of pm2 multiple copies has been reported from Mozambique [36].

In this study, few mild adverse events (a cough, fever, diarrhoea, vomiting and others) were reported and almost all resolved soon after completion of the treatment. Similar tolerable adverse events have been associated with ACT, the most common being cough, fever, vomiting followed by gastrointestinal disturbances $[15,37]$.

\section{Limitation of the study}

Although this study attained sample size below 50, minimum sample to give interpretable results, in Kibaha, Muheza, Chamwino, Kyela in 2011/2012, the results from the remaining sites show that ASAQ, AL and DHAPQ are highly effective in the treatment of uncomplicated falciparum malaria Mainland Tanzania.

Several study sites failed to enroll the target sample size of 73 cases per site per drug and four of the sites enrolled the minimum 50 cases, a sample which provides interpretable outcome. The low number of enrollments in 2011 and 2012 may be explained by the variation in seasonality and temporal downturn of malaria cases in most parts of Tanzania Mainland although resurgence was recorded afterwards from 2013 onwards [38, 39].

A large number of D0 filter paper blood samples were not available, either lost or used for parasite genotyping to differentiate reinfection from recrudescence. In addition a substantial number of the available blood spots gave either negative PCR results or non-interpretable sequences, likely due to the low amount of parasite DNA or the degradation of DNA during storage or both as samples collected in 2011-2015 were analysed end of 2017.

\section{Conclusion}

The findings documented that all the tested ACT medicines were highly efficacious, including AL despite its introduction for more than a decade, and lack of $k 13$ validated mutants. However, the presence of parasites with $p m 2$ gene amplification among the limited samples tested successfully calls for further investigations. Lastly, the population selected in this study represents the high to low transmission areas in mainland Tanzania and the most vulnerable age group for uncomplicated malaria cases.

\section{Abbreviations}

ACT: artemisinin-based combination therapy; ACPR: adequate clinical and parasitological response; AL: artemether/lumefantrine; ASAQ: artesunate/amodiaquine; DHAPQ: dihydroartemisinin/piperaquine; DNA: deoxyribonucleic acid; ETF: early treatment failure; IHI: Ifakara Health Institute; LCF: late clinical failure; LPF: late parasitological failure; mdr1: Plasmodium falciparum multi drug resistance 1; MRCC: Medical Research Coordinating Committee; NMCP: National Malaria Control Programme; PCR: polymerase chain reaction; SNP: singlenucleotide polymorphism; WHO: World Health Organization.

\section{Authors' contributions}

MAK participated in conducting the study, including; data collection, patient care and preparation of the manuscript. AMK contributed in the study design, conducting the study (overseeing research activities) and preparation of the 
manuscript. MW contributed to the design of the study, validation and data analysis and preparation of the manuscript. MKM conducted the study (supervision, patient care and data collection) and manuscript preparation. DSI, BN, $Z P, E K, M M L, C I M$ contributed in the study design, conducting the study (leading and overseeing sites specific research activities) and manuscript preparation. RM, SM and FC coordinated all research activities and participated in the manuscript preparation. DM contributed in the analysis of the K13 and pfpm2 markers, and manuscript preparation. All other authors contributed in the manuscript preparations and approved the final manuscript for publication. All authors read and approved the final manuscript.

\section{Author details}

${ }^{1}$ Ifakara Health Institute, Dar es Salaam, Tanzania. ${ }^{2}$ National Institute for Medical Research, Tanga Research Centre, Tanga, Tanzania. ${ }^{3}$ Department of Parasitology, School of Public Health, Muhimbili University of Health and Allied Sciences, Dar es Salaam, Tanzania. ${ }^{4}$ Catholic University of Health and Allied Sciences/Bugando Medical Centre, Mwanza, Tanzania. ${ }^{5}$ National Malaria Control Programme (NMCP), Dar es Salaam, Tanzania. ${ }^{6}$ World Health Organization Country Office, Dar es Salaam, Tanzania. ${ }^{7}$ Aga Khan University Hospital, Nairobi, Kenya. ${ }^{8}$ Gothenburg University, Gothenburg, Sweden. ${ }^{9}$ Institut Pasteur, Paris, France.

\section{Acknowledgements}

We would like to express gratitude to the study patients, and children who participated in the study and their parents and guardians. The staff of the sentinel sites; Chamwino, Kibaha, Kyela, Muheza, Nagaga, Rufiji, Kilombero, Butimba and Ujiji for the help and support during the study period. We would also like to thank Nimol Khim (Institut Pasteur in Cambodia) and Eric Legrand (Institut Pasteur, Paris, France) for their support in the analysis of the parasite genotyping and molecular markers.

\section{Competing interests}

The authors declare that they have no competing interests.

\section{Availability of data}

The data set used in this study is available and can be shared upon reasonable request to the corresponding author.

\section{Disclaimer}

Marian Warsame was and Ritha Njau is a staff member of the World Health Organization, and they alone are responsible for the views expressed in this publication and do not necessarily represent the decisions, policy or views of the World Health Organization.

\section{Ethics approval and consent to participate}

This study was approved by the Tanzania national ethics committee and institutions' ethics committees, and by the WHO research ethics review committee for the 2015 study. All study participants signed informed consents before enrollment.

\section{Funding}

This study was funded by the US, President's Malaria Initiative (PMI) USAID, and the World Health Organization (WHO).

\section{Publisher's Note}

Springer Nature remains neutral with regard to jurisdictional claims in published maps and institutional affiliations.

Received: 20 April 2018 Accepted: 9 October 2018

Published online: 17 October 2018

\section{References}

1. WHO. World malaria report 2017. Geneva: World Health Organization; 2017

2. $\mathrm{MOH}$. Tanzania Ministry of Health and Social Welfare. National guidelines for diagnosis and treatment of malaria; 2006

3. Dondorp AM, Nosten F, Yi P, Das D, Phyo AP, Tarning J, et al. Artemisinin resistance in Plasmodium falciparum malaria. N Engl J Med. 2009:361:455-67.
4. Leang R, Taylor WRJ, Bouth DM, Song L, Tarning J, Char MC, et al. Evidence of Plasmodium falciparum malaria multidrug resistance to artemisinin and piperaquine in western Cambodia: dihydroartemisinin-piperaquine open-label multicenter clinical assessment. Antimicrob Agents Chemother. 2015;59:4719-26.

5. WHO. Global report on antimalarial drug efficacy and drug resistance: 2000-2010. Geneva: World Health Organization; 2010.

6. Shayo A, Buza J, Ishengoma DS. Monitoring of efficacy and safety of artemisinin-based anti-malarials for treatment of uncomplicated malaria: a review of evidence of implementation of anti-malarial therapeutic efficacy trials in Tanzania. Malar J. 2015;14:135.

7. Ndounga M, Mayengue PI, Casimiro PN, Koukouikila-Koussounda F, Bitemo M, Matondo BD, et al. Artesunate-amodiaquine versus artemether-lumefantrine for the treatment of acute uncomplicated malaria in Congolese children under 10 years old living in a suburban area: a randomized study. Malar J. 2015;14:423.

8. Dambe R, Sande J, Ali D, Chilima B, Dodoli W, Michelo C, et al. Monitoring the efficacy of artemether-lumefantrine for the treatment of uncomplicated malaria in Malawian children. Malar J. 2015;14:175.

9. de Wit M, Funk AL, Moussally K, Nkuba DA, Siddiqui R, Bil K, et al. In vivo efficacy of artesunate-amodiaquine and artemether-lumefantrine for the treatment of uncomplicated falciparum malaria: an open-randomized, non-inferiority clinical trial in South Kivu, Democratic Republic of Congo. Malar J. 2016;15:455.

10. Nega D, Assefa A, Mohamed H, Solomon H, Woyessa A, Assefa Y, et al. Therapeutic efficacy of artemether-lumefantrine $\left(\right.$ Coartem $\left.^{\circledR}\right)$ in treating uncomplicated P. falciparum Malaria in Metehara, Eastern Ethiopia: regulatory clinical study. PLoS ONE. 2016;11:e0154618.

11. Ogutu BR, Onyango KO, Koskei N, Omondi EK, Ongecha JM, Otieno GA, et al. Efficacy and safety of artemether-lumefantrine and dihydroartemisinin-piperaquine in the treatment of uncomplicated Plasmodium falciparum malaria in Kenyan children aged less than five years: results of an open-label, randomized, single-centre study. Malar J. 2014;13:33.

12. Paczkowski M, Mwandama D, Marthey D, Luka M, Makuta G, Sande J, et al. In vivo efficacy of artemether-lumefantrine and artesunate-amodiaquine for uncomplicated Plasmodium falciparum malaria in Malawi, 2014. Malar J. 2016;15:236.

13. Hamainza B, Masaninga F, Moonga H, Mwenda M, Chanda-kapata P, Chalwe $V$, et al. Therapeutic efficacy of artemether-lumefantrine on treatment of uncomplicated Plasmodium falciparum mono-infection in an area of high malaria transmission in Zambia. Malar J. 2014;13:430.

14. Yeka A, Lameyre V, Afizi K, Fredrick M, Lukwago R, Kamya MR, et al. Efficacy and safety of fixed-dose artesunate-amodiaquine vs. artemetherlumefantrine for repeated treatment of uncomplicated malaria in Ugandan children. PLoS ONE. 2014;9:e113311.

15. Ursing J, Rombo L, Rodrigues A, Kofoed P-E. Artemether-lumefantrine versus dihydroartemisinin-piperaquine for treatment of uncomplicated Plasmodium falciparum malaria in children aged less than 15 years in Guinea-Bissau —an open-label non-inferiority randomized clinical trial. PLOS ONE. 2016;11:e0161495.

16. Ariey F, Witkowski B, Amaratunga C, Beghain J, Langlois AC, Khim N, et al. A molecular marker of artemisinin-resistant Plasmodium falciparum malaria in Cambodia. Nature. 2014;505:50-5.

17. Witkowski B, Duru V, Khim N, Ross LS, Saintpierre B, Beghain J, et al. A surrogate marker of piperaquine-resistant Plasmodium falciparum malaria: a phenotype-genotype association study. Lancet Infect Dis. 2017;17:174-83.

18. WHO. Methods for surveillance of antimalarial drug efficacy. Geneva: World Health Organization; 2009.

19. WHO. Guidelines for the treatment of malaria. Geneva: World Health Organization; 2010

20. Canier L, Khim N, Kim S, Sluydts V, Heng S, Dourng D, et al. An innovative tool for moving malaria PCR detection of parasite reservoir into the field. Malar J. 2013;12:405.

21. WHO. Methods and techniques for clinical trials on antimalarial drug efficacy: genotyping to identify parasite populations. Geneva: World Health Organization; 2008.

22. Malmberg M, Ngasala B, Ferreira PE, Larsson E, Jovel I, Hjalmarsson A, et al. Temporal trends of molecular markers associated with artemetherlumefantrine tolerance/resistance in Bagamoyo district, Tanzania. Malar J. 2013;12:103. 
23. Thomsen TT, Ishengoma DS, Mmbando BP, Lusingu JP, Vestergaard LS, Theander TG, et al. Prevalence of single nucleotide polymorphisms in the Plasmodium falciparum multidrug resistance gene (Pfmdr-1) in Korogwe District in Tanzania before and after introduction of artemisinin-based combination therapy. Am J Trop Med Hyg. 2011;85:979-83.

24. Sow D, Ndiaye JL, Sylla K, Ba MS, Tine RCK, Faye B, et al. Evaluation of the efficacy and safety of three 2-drug combinations for the treatment of uncomplicated Plasmodium falciparum malaria in Senegal: artesunateamodiaquine, dihydroartemisinin-piperaquine, and artemether-lumefantrine. Med Sante Trop. 2016;26:45-50.

25. Abuaku BK, Mensah BA, Ofori MF, Myers-Hansen J, Derkyi-Kwarteng AN,

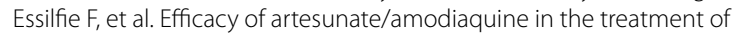
uncomplicated malaria among children in Ghana. Am J Trop Med Hyg. 2017;97:690-5

26. Wanzira H, Kakuru A, Arinaitwe E, Bigira V, Muhindo M, Conrad M, et al. Longitudinal outcomes in a cohort of Ugandan children randomized to artemether-lumefantrine versus dihydroartemisinin-piperaquine for the treatment of malaria. Clin Inf Dis. 2014;59:509-16.

27. Agarwal A, McMorrow M, Onyango P, Otieno K, Odero C, Williamson J, et al. A randomized trial of artemether-lumefantrine and dihydroartemisinin-piperaquine in the treatment of uncomplicated malaria among children in western Kenya. Malar J. 2013;12:254.

28. Plucinski MM, Talundzic E, Morton L, Dimbu PR, Macaia AP, Fortes F, et al. Efficacy of artemether-lumefantrine and dihydroartemisinin-piperaquine for treatment of uncomplicated malaria in children in Zaire and Uíge provinces, Angola. Antimicrob Agents Chemother. 2015;59:437-43.

29. WHO. Artemisinin and artemisinin-based combination therapy resistance. Geneva: World Health Organization; 2017.

30. Menard D, Khim N, Beghain J, Adegnika A, Shafiul-Alam M, Amodu O, et al. A world wide map of Plasmodium falciparum K13-propeller polymorphisms. N Engl J Med. 2016;374:2453-64.
31. Madamet M, Kounta M, Wade K, Lo G, Diawara S, Fall M, et al. Absence of association between polymorphisms in the K13 gene and the presence of Plasmodium falciparum parasites at day 3 after treatment with artemisinin derivatives in Senegal. Int J Antimicrob Agents. 2017;49:754-6.

32. Menard S, Tchoufack J, Maffo C, Nsango S, Abate L, Tsapi M, et al. Insight into k13-propeller gene polymorphism and ex vivo DHA-response profile from Cameroonian isolates. Malar J. 2016;15:572.

33. Balikagala B, Mita T, Ikeda M, Sakurai M, Yatsushiro S, Takahashi N, et al. Absence of invivo selection for K13 mutations after artemether-lumefantrine treatment in Uganda. Malar J. 2017;16:23.

34. Ljolje D, Dimbu PR, Kelley J, Goldman I, Nace D, Macaia A, et al. Prevalence of molecular markers of artemisinin and lumefantrine resistance among patients with uncomplicated Plasmodium falciparum malaria in three provinces in Angola, 2015. Malar J. 2018;17:84.

35. Amato R, Lim P, Miotto O, Amaratunga C, Dek D, Pearson RD, et al. Genetic markers associated with dihydroartemisinin-piperaquine failure in Plasmodium falciparum malaria in Cambodia: a genotype-phenotype association study. Lancet Infect Dis. 2017;17:164-73.

36. Gupta H, Macete E, Bulo H, Salvador C, Warsame M, Carvalho E, et al. Drug-resistance polymorphisms and copy numbers in Plasmodium falciparum, Mozambique, 2015. Emerg Inf Dis. 2018;24:40-8.

37. Adjei A, Narh-Bana S, Amu A, Kukula V, Nagai R, Owusu-Age S, et al. Treatment outcomes in a safety observational study of dihydroartemisinin/ piperaquine (Eurartesim ${ }^{\circledR}$ ) in the treatment of uncomplicated malaria at public health facilities in four African countries. Malar J. 2016;15:43.

38. Tanzania commission for AIDs. HIV/AIDS and malaria indicator survey 2011-12, Tanzania. Tanzania:Tanzania commission for AIDs; 2013.

39. Demographic and health survey and malaria indicator survey 2015-2016, Dar es salaam, Tanzania. 2016.
Ready to submit your research? Choose BMC and benefit from:

- fast, convenient online submission

- thorough peer review by experienced researchers in your field

- rapid publication on acceptance

- support for research data, including large and complex data types

- gold Open Access which fosters wider collaboration and increased citations

- maximum visibility for your research: over $100 \mathrm{M}$ website views per year

At BMC, research is always in progress.

Learn more biomedcentral.com/submissions 